\title{
Filtragem Wavelet de Sinais Cardíacos através de Algoritmos Adaptativos
}

\author{
Guilherme Perin ${ }^{1}$ \\ Alice de Jesus Kozakevicius ${ }^{2}$
}

\begin{abstract}
Resumo: Neste trabalho o algoritmo de Azzalini, Farge e Schneider, utilizado em análise de imagens, é modificado, produzindo uma versão adaptativa e uma recursiva para a filtragem de sinais cardíacos. Através destes algoritmos um limiar de corte é obtido baseado na variância do ruído e a série wavelet do sinal analisado é então truncada. Wavelets ortonormais de Daubechies são consideradas. Para validação do algoritmo proposto, sinais ECG da base de dados MIT-BIH com diferentes níveis de ruído são filtrados e a medida de qualidade SNR é calculada para cada caso. As simulações mostram a performance do algoritmo proposto para diferentes intensidades de ruído, e para diferentes níveis da transformada wavelet e em comparação a outros dois métodos de filtragem.
\end{abstract}

\begin{abstract}
In this paper the image analysis algorithm proposed by Azzalini, Farge and Schneider is modified, given rise to an adaptative and a recursive version for filtering cardiac signals. These algorithms produce a threshold value based on the estimation of the noise variance and the wavelet series from the analysed signal is thresholded. Orthonormal wavelets from the Daubechies family are considered. To validate the proposed algorithm, ECG signals from the MIT-BIH data base with different noise levels are filtered and the quality measure computed is the SNR value, also based on the variance estimation. The simulation show the performance from the proposed algorithm for different levels of noise, for different levels of the wavelets transform and in comparison with two other filtering methods.
\end{abstract}

\section{Introdução}

Um eletrocardiograma (ECG) é um registro gráfico, um traçado, das atividades elétricas que ocorrem no músculo cardíaco ao longo de sua movimentação (batimentos). Neste traçado cinco formas de onda são identificadas e classificadas pelos especialistas como ondas $\mathrm{P}, \mathrm{Q}, \mathrm{R}, \mathrm{S}$ e T, Figura 1. A onda $\mathrm{P}$ representa a contração do átrio. O agrupamento central, denominado complexo QRS, representa uma grande deflexão quando os ventrículos são

\footnotetext{
${ }^{1}$ PPGI, UFSM

\{guilhermeperin7@gmail.com\}

${ }^{2}$ LANA/PPGI, UFSM

\{alicek@smail.ufsm.br\}
} 
despolarizados. $\mathrm{E}$ a onda $\mathrm{T}$ do $\mathrm{ECG}$ está associada ao retorno do ventrículo à sua posição de equilíbrio elétrico (repolarização). O complexo QRS, agrupamento de maior amplitude, é fundamental para o reconhecimento do funcionamento normal do coração. Em [12, 13] encontram-se detalhes específicos sobre a atividade cardíaca e sobre a obtenção de ECG's, cuja captação é feita através de eletrodos, capazes de registrar além das atividades elétricas do coração, demais atividades musculares do paciente, interferências elétricas inerentes à corrente elétrica e pertubações oriundas no processo de transmissão dos dados medidos. Todos essas contriuições extras capturadas ao longo do processo causam interferências no sinal de interesse e são denominadas ruído. De acordo com sua intensidade, esse ruído necessita ser removido ou atenuado para que o sinal ECG possa ser analisado e interpretado. Este trabalho tem como objetivo principal dar uma contribuição para filtragem adaptativa desse ruído através de transformada discreta wavelet. Nos últimos anos, muitos estudos têm sido realiza-

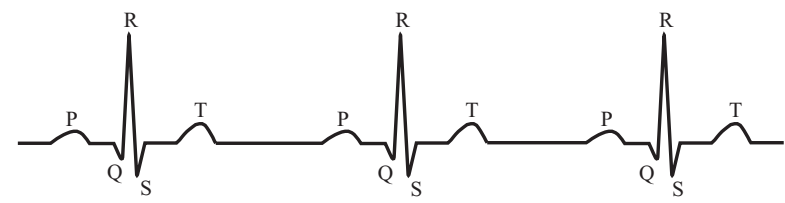

Figura 1. Sinal cardíaco normal: ondas $\mathrm{P}, \mathrm{T}$ e complexo QRS.

dos quanto à utilização de transformadas wavelet para remoção de ruído (filtragem) de sinais cardíacos (ECG) e demais sinais biomédicos. Vários métodos de filtragem destes sinais, que utilizam essas transformadas como parte do processo de filtragem, foram propostos como adaptações ao método wavelet shrinkage, desenvolvido em [2]. Este método baseia-se na determinação de um valor de corte (threshold value) para o truncamento (hard thresholding) da série wavelet de uma função, assumindo como hipótese que o ruído agregado inicialmente aos dados é do tipo gaussiano. A série wavelet do sinal poluído (função mais ruído) é então truncada levando-se em consideração o valor de corte estipulado. Através da transformada wavelet inversa aplicada a essa nova série, obtém-se o sinal filtrado.

Em [8], a técnica de filtragem apresentada para sinais cardíacos combina truncamento (hard thresholding) com estratégias de amortecimento (soft thresholding) dos coeficientes da série wavelet, cujo objetivo é corrigir os efeitos não esperados (fenômeno de Gibbs, atenuação da onda R, eliminação indesejada das ondas $\mathrm{P}$ e S) ocasionados pela aplicação de hard e soft thresholding separadamente. Porém, esse método mostrou-se eficiente apenas quando fixados 4 níveis de decomposição da transformada wavelet. Além disso, também foram fixados de forma empírica parâmetros de ponderação entre as estratégias de amortecimento e de truncamento. Em [9] é proposto um método de filtragem e deteç̧ão do complexo QRS baseado na transformada wavelet direta e em estratégias adaptativas de threshold, também fazendo referência ao método dado em [2]. O trabalho consiste em encontrar dois valores de 
corte de modo a enquadrar o complexo QRS entre tais valores.

A grande questão abordada em [10] é a dificuldade de avaliação da qualidade da filtragem realizada, não só em termos quantitativos (erro quadrático médio), mas em termos qualitativos, de tal modo que propriedades do sinal cardíaco, essenciais em diagnósticos, não sejam modificadas, distorcidas ou removidas do sinal analisado. No entanto, a dificuldade em aplicar medidas de qualidade que levem em consideração propriedades morfológicas do sinal (duração da onda $\mathrm{P}$, tamanho do intervalo QT, forma da onda T, elevação da onda $\mathrm{S}$ até T) está na obtenção de um banco de dados para a forma padrão do sinal e suas variações morfológicas de acordo com anomalias e/ou patologias cadastradas, indivíduos de diferentes faixas etárias, com biotipos distintos, etc. Trabalhos como o desenvolvido em [10] consideram bases de dados de acesso restrito e pago, o que dificulta (ou invibializa) a reprodução de seus resultados. Como uma alternativa para este contexto, a base de dados MIT-BIH [6] é uma das bases de dados biomédicos de acesso gratuito mais renomadas para obtenção de sinais ECG, da qual foram considerados todos os sinais testados neste trabalho. Esta base fornece 48 trechos de meia hora de dois canais de ECG's obtidos de 47 indivíduos estudados entre 1975 e 1979. Vinte e três gravações foram escolhidas aleatoriamente a partir de um conjunto de 4000 ECG's coletados em exames de $24 \mathrm{~h}$ de duração de uma população mista de pacientes. As gravações, digitalizadas em 360 amostras por segundo por canal, com 11 bits de resolução sobre uma faixa de $10 \mathrm{mV}$, são apropriadas para estudos de diferentes arritmias e tópicos relacionados [6].

O presente trabalho, seguindo a linha das contribuições de [9, 8], tem por objetivo a aplicação e análise de dois algoritmos baseados em transformada wavelet discreta de Daubechies, para encontrar um valor de corte que seja adequado para operações de truncamento (hard thresholding) de modo que o ruído, causado por interferências do ambiente e pelo equipamento de medição do ECG, possa ser eliminado sem afetar a integridade do sinal original. O primeiro algoritmo apresentado é denominado AFS-Modificado por ser inspirado no algoritmo apresentado por Azzalini, Farge e Schneider [1]. A modificação proposta é baseada no espectro de frequências do sinal e faz uso da aplicação da técnica de hard thresholding. Diferentemente de [8], a filtragem através deste algortimo não sofre efeitos significativos em função da quantidade de níveis da transformada wavelet direta, pois o limiar de corte é obtido adaptativamente através dos dados do sinal. O segundo algoritmo proposto é uma adaptação a sinais unidimensionais do método apresentado em [1]. Novamente a série wavelet do sinal é truncada através da estratégia de hard thresholding. A diferença entre os algoritmos está na maneira com que o limiar de corte é obtido. Nesta segunda proposta, o algoritmo trabalha de maneira recursiva e tem por objetivo encontrar um valor de corte ótimo, que minimize o número de coeficientes truncados.

Este trabalho está organizado da seguinte maneira: a Seção 2 apresenta um breve resumo sobre transformada wavelet. As Seções 3 e 4 apresentam as modificações propostas 
para os algoritmos de filtragem AFS original, denominados algoritmos adaptativo e recursivo. Na Seção 5 é apresenta, através da relação sinal ruído, uma maneira de se analisar a qualidade da filtragem. E finalmente na Seção 6 são apresentadas algumas aplicações dos algoritmos a sinais cardíacos do banco de dados MIT-BIH[6].

\section{Transformada Wavelet e Thresholding}

O ambiente natural para a teoria das funções wavelets é chamado de análise de multiresolução, que consiste na construção de espaços encaixados $V_{j}$ gerados por estas funções, suas translações e dilatações [7]. Um dos axiomas principais assume que $\{0\} \subset \ldots \subset V_{-1} \subset$ $V_{0} \subset V_{1} \subset \ldots \subset \mathcal{L}^{2}(\mathcal{R})$, portanto as funções wavelets formam uma base para representação de funções de energia finita, $f \in \mathcal{L}^{2}(\mathcal{R})$.

Neste trabalho considera-se a família de wavelets ortonormais de Daubechies, que são funções não nulas apenas em um intervalo fechado da reta (suporte compacto). A família de funções é gerada por $\phi(x)$, dita função escala, e por $\psi(x)$, a função wavelet correspondente, ortogonal a $\phi(x)$. Os espaços $V_{j}$ são gerados pelas translações das funções escalas, $V_{j}=$ $\left[\phi_{j, k}(x) / j, k \in \mathcal{Z}\right] \operatorname{com} \phi_{j, k}(x)=2^{j / 2} \phi\left(2^{j} x-k\right)$ para $j, k \in \mathcal{Z}$. Aqui $j$ indica a escala, ou nível de resolução, que está associada ao espaçamento dos pontos do stencil de representação dos dados em cada espaço $V_{j}$, e $k$ é o parâmetro de translação dentro de cada nível $j$.

Analogamente $\psi_{j, k}(x)=2^{j / 2} \psi\left(2^{j} x-k\right)$, para $j, k \in \mathcal{Z}$ geram os espaços $W_{j}=$ $\left[\psi_{j, k}(x) / j, k \in \mathcal{Z}\right]$ ortogonais a $V_{j}$. Além disso, para todo nível $j$ de resolução, vale a seguinte relação $V_{j+1}=V_{j} \oplus W_{j}$, em que $\oplus$ representa a soma direta. Isso significa que os dados representados em $V_{j+1}$ são decompostos em dados com um nível a menos de resolução, contidos em $V_{j}$ e $W_{j}$, cujas componentes de $V_{j}$ independem das componentes de $W_{j}$. Considerando-se ainda $J$ o nível de resolução mais fino e $J_{0}$ o nível de resolução mais grosseiro, tem-se $V_{J}=V_{J_{0}} \oplus\left(\bigoplus_{j=J_{0}}^{J-1} W_{j}\right)$. Assim, considerando $J_{0} \rightarrow-\infty$ e $J \rightarrow \infty$, obtém-se $\mathcal{L}^{2}(\mathcal{R})=\bigoplus_{j=-\infty}^{\infty} W_{j}$ e $\bigcap_{j=-\infty}^{\infty} V_{j}=\{0\}$. As funções escala e wavelet desta família satisfazem as seguintes relações, ditas relações de escala:

$$
\phi_{j-1, l}(x)=\sum_{k=0}^{D-1} a_{k} \phi_{j, 2 l+k}(x) \text { e } \psi_{j-1, l}(x)=\sum_{k=0}^{D-1} b_{k} \phi_{j, 2 l+k}(x),
$$

cujos filtros $a_{k}$ e $b_{k}=(-1)^{k} a_{D-1-k}$ caracterizam as funções da família de Daubechies [3], sendo $D=2 N$ e $N$ representa o número de momentos nulos da wavelet escolhida. Considerando $f \in \mathcal{L}^{2}(\mathcal{R})$, a projeção desta função em $V_{j}$ é dada por:

$$
\left(P_{V_{j}} f\right)(x)=\sum_{l=-\infty}^{\infty} c_{j, l} \phi_{j, l}(x), \text { sendo } c_{j, l}=\int_{-\infty}^{\infty} f(x) \phi_{j, l}(x) d x .
$$


Os coeficientes de escala $c_{j, l}$ são dados pelo produto interno da função $f$ com as funções escala $\phi_{j, l}(x)$. Como $W_{j-1}$ é complemento ortogonal de $V_{j-1}$ em $V_{j}$, tem-se ainda $\left(P_{V_{j}} f\right)(x)=$ $\left(P_{V_{j-1}} f\right)(x)+\left(P_{W_{j-1}} f\right)(x)$, sendo a projeção $\left(P_{W_{j-1}} f\right)(x)$ dada como em 2 com coeficientes wavelets denotados por $d_{j-1, l}$ e obtidos através do produto interno da função $f$ e as funções wavelets $\psi_{j, l}(x)$. Portanto a projeção da $f$ em $V_{j}$ pode ser dada pela formulação abaixo em termos das funções escala e wavelet envolvendo coeficientes $c_{j-1, k}$ e $d_{j-1, k}$, ambos dados na escala $j-1$.

$$
\left(P_{V_{j}} f\right)(x)=\sum_{l=-\infty}^{\infty} c_{j-1, l} \phi_{j-1, l}(x)+\sum_{l=-\infty}^{\infty} d_{j-1, l} \psi_{j-1, l}(x) .
$$

Observe que para uma escala $j$ suficientemente fina, $\phi_{j}$ diferente de zero apenas em um intervalo fechado da reta muito pequeno, portanto os coeficientes $c_{j, k}, \forall k$ podem ser aproximados pelos valores da função a ser analisada em pontos de uma malha fixada na escala $j$ :

$$
c_{j, k}=\int_{-\infty}^{\infty} f(x) \phi_{j, k}(x) d x \approx f\left(x_{j, k}\right) .
$$

O objetivo desta observação é estabelecer uma relação entre a seqüência de coeficientes $\left\{c_{j, l}\right\}_{l \in Z}$ e as sequiências $\left\{c_{j-1, l}\right\}_{l \in Z}$ e $\left\{d_{j-1, l}\right\}_{l \in Z}$, de tal modo que apenas os filtros $a_{k}$ e $b_{k}$ sejam utilizados, evitando cálculo explícito das funções wavelet e escala e evitando também o cálculo dos coeficientes $c_{j, k}$ no nível mais fino de resolução através da definição apresentada em (2). Desta forma, substituindo-se a relação (1) na definição de $c_{j, l}$, tem-se:

$$
c_{j-1, l}=\int_{-\infty}^{\infty} f(x) \sum_{k=0}^{D-1} a_{k} \phi_{j, 2 l+k}(x) d x=\sum_{k=0}^{D-1} a_{k} c_{j, 2 l+k} .
$$

Analogamente relacionam-se os coeficientes $d_{j-1, l}$ com os $d_{j, k}$ e os filtros $b_{k}$. Estas relações definem a Transformada Rápida Wavelet [3], cujos dados iniciais são aproximados apenas para o nível mais fino de resolução por (4). $\mathrm{O}$ algoritmo rápido para a transformada wavelet direta (TWD) é dado por:

$$
c_{j-1, l}=\sum_{k=0}^{D-1} a_{k} c_{j, 2 l+k} \text { e } d_{j-1, l}=\sum_{k=0}^{D-1} b_{k} c_{j, 2 l+k} .
$$

Esta transformação define um mapeamento linear dos coeficientes $\left\{c_{j, k}\right\}_{k \in Z}, j$ nível mais fino, em $\left\{c_{j-1, k}\right\}_{k \in Z}$ e $\left\{d_{j-1, k}\right\}_{k \in Z}$. A fatoração de funções no espaço $V_{j}$ em uma quantidade maior de níveis é obtida através da aplicação da mesma transformação na sequiência $\left\{c_{j-1, l}\right\}_{l \in Z}$ para obter as novas seqüências $\left\{c_{j-2, l}\right\}_{l \in Z}$ e $\left\{d_{j-2, l}\right\}_{l \in Z}$ e assim sucessivamente, até que o nível grosseiro $J_{0}$ seja alcançado. Aqui $J_{0}$ tanto pode ser um nível intermediário desejado, quanto pode ser o nível mais grosseiro contendo apenas um elemento em 
cada componente. Sendo assim, pode-se representar esta transformada entre vários níveis através da seguinte fatoração, considerando $j=J, J-1, \ldots, J_{0}$.

$$
f(x)=\sum_{l=-\infty}^{\infty} c_{J_{0}, l} \phi_{J_{0}, l}(x)+\sum_{j=J_{0}}^{J-1} \sum_{l=-\infty}^{\infty} d_{j, l} \psi_{j, l}(x), x \in \mathbf{R} .
$$

Para funções (sinais) cuja discretização é feita apenas para um intervalo limitado do domínio e não para a reta toda, os somatórios em (7) passam a ser finitos, $0 \leq l \leq N_{j}$, com $N_{j}=2^{j}$ $\left(j=J_{0}, J-1, \ldots, J\right)$ sendo o número de pontos do nível considerado (ou no caso finito $\left\{d_{j, l}\right\}, 0 \leq l \leq N_{j}$ ). Uma vez que os elementos $\left\{d_{j, l}\right\}_{l \in Z}$ tenham sido calculados, eles não serão modificados nos passos subsequentes, o que torna a transformada wavelet rápida muito eficiente em termos de implementação computacional. A transformada inversa é obtida de maneira similar, quando se substitui as relações de escala na expressão (3):

$$
\begin{gathered}
\sum_{l=-\infty}^{\infty} c_{j, l} \phi_{j, l}(x)=\sum_{n=-\infty}^{\infty} c_{j-1, n} \phi_{j-1, n}(x)+\sum_{n=-\infty}^{\infty} d_{j-1, n} \psi_{j-1, n}(x), \\
\left.\sum_{l=-\infty}^{\infty} c_{j, l} \phi_{j, l}(x)=\sum_{n=-\infty}^{\infty} c_{j-1, n} \sum_{k=0}^{D-1} a_{k} \phi_{j, 2 n+k}(x)+\sum_{n=-\infty}^{\infty} d_{j-1, n} \sum_{k=0}^{D-1} b_{k} \psi_{j, 2 n+k}(x), x\right) \\
\sum_{l=-\infty}^{\infty} c_{j, l} \phi_{j, l}(x)=\sum_{k=0}^{D-1} \sum_{n=-\infty}^{\infty}\left[c_{j-1, n} a_{k}+d_{j-1, n} b_{k}\right] \phi_{j, 2 n+k}(x) .
\end{gathered}
$$

No entanto, são introduzidas as variáveis $l=2 n+k$ na última expressão. Desde que $k=$ $l-2 n$ e $k \in[0, D-1]$, encontramos, para um dado valor para a posição $l$ os seguintes limites em $n:\left\lceil\frac{l-D+1}{n}\right\rceil \equiv n_{1}(l) \leq n \leq n_{2}(l) \equiv\left\lfloor\frac{l}{2}\right\rfloor$. Assim:

$$
\sum_{l=-\infty}^{\infty} c_{j, l} \phi_{j, l}(x)=\sum_{n=-\infty}^{\infty} \sum_{n=n_{1}(l)}^{n_{2}(l)}\left[c_{j-1, n} a_{l-2 n}+d_{j-1, n} b_{l-2 n}\right] \phi_{j, l}(x) .
$$

E, calculando os coeficientes, obtem-se a transformada wavelet inversa (TWI):

$$
c_{j, l}=\sum_{n=n_{1}(l)}^{n_{2}(l)} c_{j-1, n} a_{l-2 n}+d_{j-1, n} b_{l-2 n} .
$$

Esta é uma transformada wavelet inversa parcial, pois envolve apenas dois níveis consecutivos. Consequentemente, a transformada wavelet rápida inversa é obtida pela aplicação da repetição da relação (12) para todos os níveis de fatoração $j=J_{0}+1, J_{0}+2, \ldots, J$.

\subsection{Operação de Truncamento}

A propriedade de possuir $N$ momentos nulos das wavelets de Daubechies tem como consequência principal o fato de, para uma função suave, os coeficientes wavelets $\left\{d_{j, k}\right\}_{k \in Z}$ decrescerem rapidamente [7]. Além disso, se uma função possuir descontinuidades em uma 
de suas derivadas, então os coeficientes wavelets de sua série irão decrescer mais lentamente apenas em uma vizinhança desta descontinuidade, permanecendo com decaimento rápido em regiões nas quais a função original é suave. Uma aplicação imediata disso é a possibilidade de se descartar coeficientes wavelet que sejam menores do que um certo valor de corte, sem perda significativa de "qualidade"do sinal, quando este é recuperado após a transformada inversa da série truncada. Aqui cabe salientar que este é o princípio fundamental por trás da compressão e análise de dados usando transformadas wavelet.

Na filtragem de sinais (biomédicos ou não), cuja técnica envolva aplicação de transformada wavelet associada à operação de truncamento de coeficientes wavelets da série, duas estratégias de truncamento (thresholding) são muito utilizadas: as estratégias de hard thresholding e de soft thresholding [5, 8]. Ambas necessitam da obtenção prévia de um limiar de corte $\lambda$ (threshold value) que, aplicado aos coeficientes da série wavelet de uma função, possa selecionar informações de acordo com a suavidade do sinal analisado. A estratégia de Hard Thresholding está definida por:

$$
T h_{H}(d)=\left\{\begin{array}{lll}
d, & \text { se } & (|d| \geq \lambda) \\
0, & \text { se } & (|d|<\lambda) ; \quad d=d_{j k}, \forall j, \forall k .
\end{array}\right.
$$

Neste trabalho, o foco central é a determinação do limiar de corte $\lambda$ para que, a partir dele, ruídos de alta frequência (informações expúrias) dos sinais cardíacos possam ser retirados. Em todos os experimentos numéricos considera-se a estratégia de Hard Thresholding, pois a operação de truncamento com esta estratégia tende a manter a forma da onda $\mathrm{P}$, do complexo QRS e da onda $\mathrm{T}$ do sinal cardíaco quase sem alterações, como indicam os experimentos numéricos apresentados na próxima seção.

\section{O Algoritmo Adaptativo AFS - Modificado}

Donoho em [2] propõe uma técnica de filtragem de coeficientes da série wavelet para um sinal original sendo considerado como $y=s+e$, ou seja, $y$ representado pela soma do sinal puro $s$ com um ruído gaussiano $e$. A técnica proposta em [2] está fortemente baseada na hipótese de $e$ ser um ruído gaussiano, cuja estimativa da variância do ruído é feita através desta hipótese. O limiar de corte $\lambda$ é então determinado por Donoho através da variância do ruído gaussiano, cuja expressão é dada por: $\lambda_{U}=\sigma \sqrt{2 \log _{10} N}$. Este valor é denominado pelo autor como universal threshold.

No contexto de sinais biomédicos (cardíacos), ao contrário dos sinais analisados em [2], a componente de ruído é uma somatória de várias interferências que ocorrem durante a captação do sinal, e por consequência, de natureza desconhecida. Sendo assim, técnicas adaptativas para determinação dos valores de truncamento da série wavelet para sinais cardíacos 
têm apresentado resultados promissores tanto em processos de filtragem [5], quanto em processos de análise de componentes específicas do sinal, como por exemplo, complexo QRS, ondas P e T [8, 9, 6]. Com o intuito de contribuir nessa linha, este trabalho descreve modificações e o uso de um algoritmo adaptativo que tem por objetivo estimar a variância da componente de ruído presente no sinal analisado e, através disso, obter um coeficiente de corte $\lambda$ que seja coerente com este sinal.

Novamente considera-se $y$ como sendo o sinal com ruído, $s$ o sinal puro a ser estimado após a inversão da série wavelet truncada e $e$ o ruído presente em $y$. $\mathrm{O}$ algoritmo adaptativo possui as etapas de inicialização e filtragem. Para simplificar a notação, considera-se o vetor dos coeficientes wavelet por nível $d_{j}=\left\{d_{j, k}\right\}_{k \in Z}, \forall j$ :

\section{O Algoritmo Adaptativo AFS - Modificado:}

\section{Etapa 1: Inicialização:}

1.1. $\left(y_{1}, d_{1}\right) \leftarrow T W D\left(y_{0}\right)$ : Aplicar um nível da TWD ao sinal original $y_{0}$, obtendo $y_{1}$ que representa o sinal grosseiro e $d_{1}$ que representa um sinal de detalhes, ambos vetores com $N / 2$ pontos no nível $n_{1}$;

1.2. $\sigma_{d_{1}}$ : Calcular o desvio padrão do sinal de detalhes $d_{1}$, obtido no passo 1.1 ;

1.3. $\lambda_{d_{1}}$ : Calcular o coeficiente de corte $\lambda_{d_{1}}=\frac{1}{2} \sigma_{d_{1}} \sqrt{2 \log _{10}(N / 2)}$. O número do pontos no cálculo de $\lambda_{d_{1}}$ é $N / 2$ ao invés de $N$, como apresentado em [2], e também dividido por 2. Isso diminui a magnitude do coeficiente de corte, o que resulta na preservação de informações importantes do sinal cardíaco;

\section{Etapa 2: Filtragem:}

2.1. $\left(y_{n}, d_{n}, d_{n-1}, \ldots, d_{2}\right) \leftarrow T W D\left(y_{1}\right)$ : Aplicar TWD para $n$ níveis ao vetor $y_{1}$. Cada vetor $d_{j}$ tem metade dos elementos de $d_{j-1}$;

2.2. $\tilde{d}_{1} \leftarrow(0,0, \ldots, 0)$ e $\left(\tilde{d}_{n}, \tilde{d}_{n-1}, \ldots, \tilde{d}_{2}\right) \leftarrow T h_{H}\left(d_{n}, d_{n-1}, \ldots, d_{2}\right)$ : Descartar todos os coeficientes $d_{1}$ e aplicar a operação de truncamento $\operatorname{com} \lambda=\lambda_{d_{1}}$ aos vetores dos coeficientes wavelets $d_{2}, d_{3}, \ldots$, $d_{n}$, dados na etapa 2.1 ;

2.3. $y_{0}^{\text {filter }} \leftarrow T W D I\left(y_{n}, \tilde{d}_{n}, \tilde{d}_{n-1}, \ldots, \tilde{d}_{1}\right)$ : Aplicar transformada inversa aos vetores do passo 2.2 para obter $y_{0}^{\text {filter }}$, que representa o sinal filtrado.

\subsection{Operação de Truncamento x Frequências}

Neste trabalho, uma das alterações propostas é a maneira com que o valor de corte inicial para o processo de filtragem é obtido. Na versão original do algortimo apresentada em [1], todos os coeficientes wavelets de todos os níveis da série são considerados. Aqui, propomos que a primeira aproximação para o coeficiente de corte $\lambda_{d_{1}}$ seja calculada apenas através do vetor que representa o sinal de detalhes $d_{1}$, obtido a partir da transformada wavelet direta para o primeiro nível de decomposição. Isso se deve ao fato do primeiro nível da transformada conter a maior quantidade de informações de alta frequência em relação a toda a fatoração do sinal e portanto, com maior chance de representar informações associadas a 
Filtragem Wavelet de Sinais Cardíacos através de Algoritmos Adaptativos
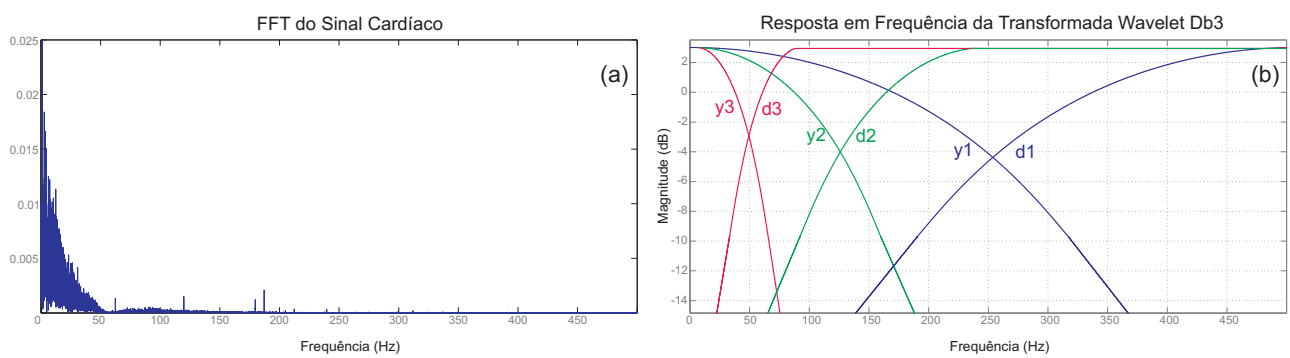

Figura 2. (a) FFT de um sinal cardíaco digitalizado com 1000 amostras por segundo. (b) Análise no domínio da frequência para a transformada wavelet direta do sinal $y_{0}$ para três níveis de decomposição. Note que $y_{1}, y_{2}$ e $y_{3}$ são as componentes de escala e $d_{1}, d_{2}$ e $d_{3}$ são as componentes wavelets dos 3 níveis.

perturbações (ruído). Como o algoritmo proposto é adaptativo, ao longo das demais iterações, novas estimativas para $\lambda$ são obtidas a partir do valor inicialmente estimado.

No caso de um sinal cardíaco ter uma taxa de amostragem de 1000 amostras por segundo, no domínio da frequência esses valores estão assossiados a um espectro de frequências de 0 a $500 \mathrm{~Hz}$, conforme ilustrado na Figura2 (a). Para sinais cardíacos, as componentes importantes se concentram entre 0 e $120 \mathrm{~Hz}$ (ondas P, Q, R, S e T) de acordo com [6]. Portanto, as informações principais do sinal concentram-se na primeira metade do espectro de frequências $[0-250 \mathrm{~Hz}]$. Já a segunda metade do espectro de frequência $[250-500 \mathrm{~Hz}]$ representa a maior parte das componentes de ruído presentes no sinal cardíaco. Isso indica que coeficientes wavelets associados a altas frequências podem ser retirados do sinal, sem danificar a estimativa do sinal sem ruído, justificando a escolha feita para nosso primeiro valor de corte.

Os filtros wavelets $a_{k}$ e $b_{k}$, separam portanto o sinal original $y_{0}$ em componentes $y_{1}$ e $d_{1}$, cujas frequências situam-se entre $[0-250 \mathrm{~Hz}]$ e $[250-500 \mathrm{~Hz}]$, respectivamente. De forma análoga, as componentes $y_{2}$ e $d_{2}$ terão suas frequências situadas em [0-125Hz] e [125-250Hz], ou seja, na primeira e segunda metades da faixa de variação correspondente à componente $y_{1}$. De forma sucessiva as demais componetes $y_{j+1}$ e $d_{j+1}$ estarão situadas na primeira e segunda metade do espectro de frequências associadas à componente $y_{j}$, como indicado na Figura 2, O gráfico apresentado na Figura 2(b) é obtido através das respostas em frequência dos filtros wavelets $a_{k}$ e $b_{k}$ (obtido via FFT dos vetores $a_{k}$ e $b_{k}$, ambos de comprimento $D$ ), como mostram as equações abaixo, sendo $\mathbf{i}=\sqrt{-1}$ e $\omega$ a frequência:

$$
A(\omega)=\sum_{k=0}^{D-1} a_{k} e^{(-\mathbf{i} \omega k)}, \quad B(\omega)=\sum_{k=0}^{D-1} b_{k} e^{(-\mathbf{i} \omega k)} .
$$


Levando em consideração o espectro de frequências de um sinal cardíaco, a Figura [2 b) mostra que para os três primeiros níveis de decomposição os vetores de detalhes $d_{1}, d_{2}$ e $d_{3}$ retêm apenas informações que representam componentes de ruído, podendo ser descartadas sem danificar as componentes significativas para a reconstrução do sinal cardíaco.

\section{O Algoritmo Recursivo AFS}

O algoritmo recursivo AFS proposto em [1] foi elaborado para análise de sinais bidimensionais (imagens). Aqui, é proposta uma adaptação deste algoritmo para sinais unidimensionais. O algoritmo AFS também baseia-se na obtenção de um coeficiente de corte ótimo através da análise de um sinal original $y=s+e, s$ representando o sinal puro e $e$, uma componente de ruído presente em $y$. A seguir as três etapas que caracterizam o algoritmo AFS são apresentadas com as adaptações necessárias para sinais unidimensionais, preservando-se a notação fixada em [1].

\section{O Algoritmo Recursivo AFS}

\section{Etapa 1: Inicialização}

1.1. $i \leftarrow 0$ : Considerar a iteração inicial com $i=0$;

1.2. $\bar{y}=\left(y_{n}, d_{n}, d_{n-1}, \ldots, d_{1}\right) \leftarrow T W D\left(y_{0}\right)$ : Aplicar a TWD ao sinal $y_{0}$, com $N$ pontos, para $n$ níveis de decomposição, obtendo $\bar{y}$, que contém os coeficientes da série wavelet $d_{1}, d_{2} \ldots d_{n}$ e $y_{n}$;

1.3. $\sigma_{\bar{y}}$ e $\lambda_{\bar{y}}$ : Calcular o desvio padrão de $\bar{y}$, e o coeficiente de corte $\lambda_{\bar{y}}=\sigma_{\bar{y}} \sqrt{2 \log _{10}(N)}$. Este desvio padrão difere da estimativa apresentada no algoritmo AFS Modificado, pois agora são tomados todos os valores da série completa de $\bar{y}$;

1.4. $\left(\tilde{d}_{n}, \tilde{d}_{n-1}, \ldots, \tilde{d}_{1}\right) \leftarrow T h_{H}\left(d_{n}, d_{n-1}, \ldots, d_{1}\right)$ : Aplicar a operação $T h_{H}$ de hard thresholding com limiar de corte dado por $\lambda_{\bar{y}}$ aos coeficientes wavelets $d_{1}, d_{2} \ldots d_{n}$, obtidos em 1.2;

1.5. $N_{i}$ : Armazenar em uma variável $N_{i}$ o número de coeficientes de detalhes que foram descartados pela operação de truncamento realizada em 1.4;

1.6. $\sigma_{i}$ : Calcular o desvio padrão $\sigma_{i}$ dos coeficientes que foram descartados em 1.4;

1.7. $\lambda_{i}$ : Calcular o limiar de corte $\lambda_{i}=\sigma_{i} \sqrt{2 \log _{10}(N)}$, obtido a partir do desvio padrão estimado em 1.6 e iniciar o laço principal:

\section{Etapa 2: Laço Principal-correção do valor inicial estimado}

2.1. $\left(\tilde{d}_{n}, \tilde{d}_{n-1}, \ldots, \tilde{d}_{1}\right)^{(i)} \leftarrow T h_{H}^{\lambda_{i}}\left(d_{n}, d_{n-1}, \ldots, d_{1}\right)$ : Aplicar a operação $T h_{H}$ com limiar $\lambda_{i}$ aos coeficientes $d_{1}, d_{2} \ldots d_{n}$ obtidos a partir da TWD de $y_{0}$;

2.2. $N_{i+1}$ : Armazenar na variável $N_{i+1}$ o número de coeficientes wavelets descartados pela operação de corte em 2.1;

2.3. $\sigma_{i+1}$ : Calcular o novo desvio padrão $\sigma_{i+1}$ de todos os coeficientes wavelets descartados em 2.1 ;

2.4. $\lambda_{i+1}$ : Calcular o novo limiar de corte $\lambda_{i+1}=\sigma_{i+1} \sqrt{2 \log _{10}(N)}$;

2.5. Se $N_{i}=N_{i+1}$, passar para a operação final. Caso contrário, $i=i+1$ e retornar ao passo 2.1;

\section{Etapa 3: Operação Final}

3.1. $\left(\tilde{d}_{n}, \tilde{d}_{n-1}, \ldots, \tilde{d}_{1}\right) \leftarrow T h_{H}\left(d_{n}, d_{n-1}, \ldots, d_{1}\right)$ : Aplicar a operação de corte com $\lambda_{i+1}$ aos coefi- 

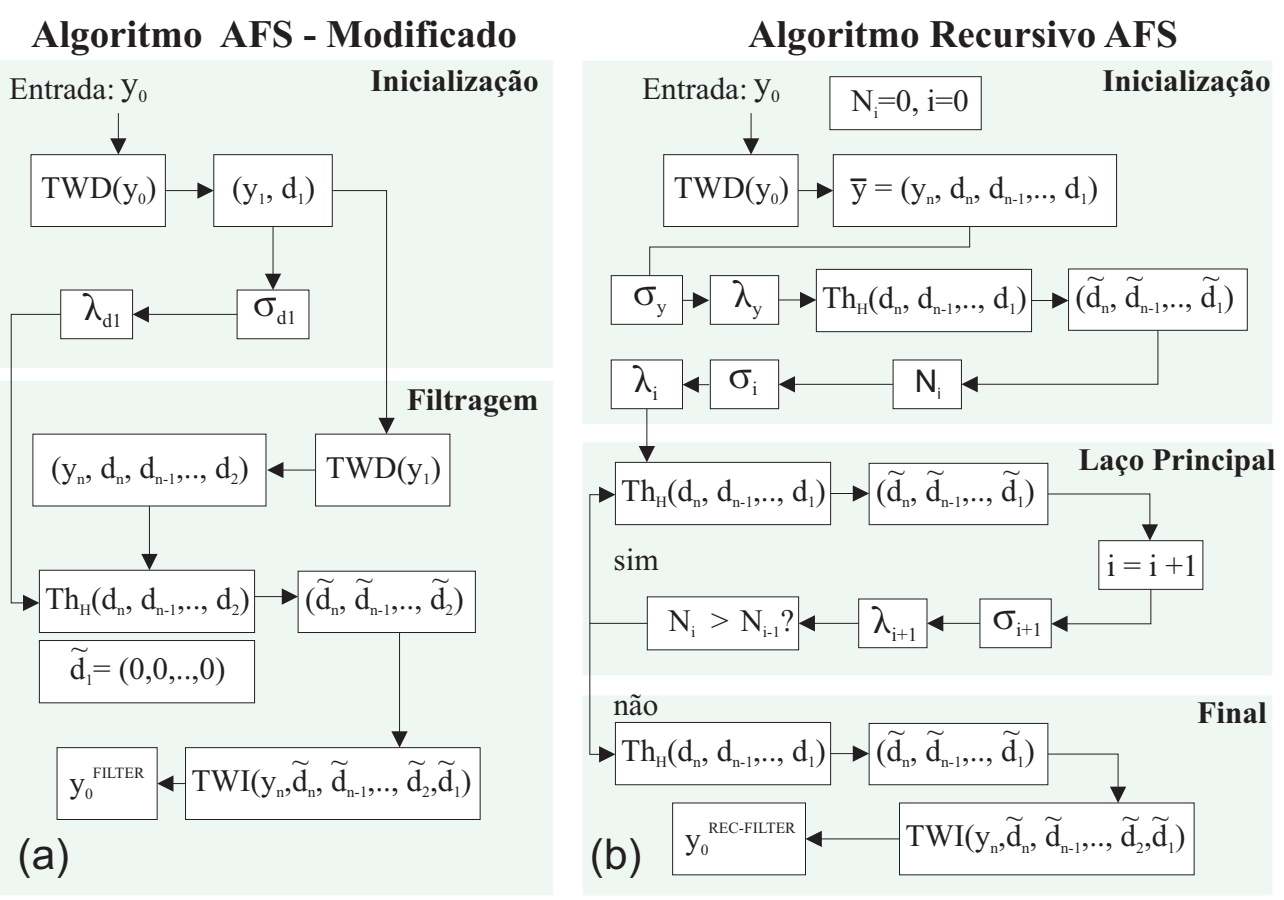

Figura 3. Fluxograma dos algoritmos (a) Adaptativo AFS Modificado e (b) Recursivo AFS.

cientes wavelets $d_{1}, d_{2} \ldots d_{n}$ obtidos a partir da TWD de $y_{0}$;

3.2. $y_{0}^{\text {rec-filter }}=T W I\left(y_{n}, \tilde{d}_{n}, \tilde{d}_{n-1}, \ldots, \tilde{d}_{1}\right)$ : Reconstruir o sinal a partir da tranformada wavelet inversa utilizando os coeficientes truncados $\tilde{d}_{1}, \tilde{d}_{2} \ldots ; \tilde{d}_{n}$ e $y_{n}$ obtidos no passo 3.1. O resultado obtido nesta etapa representa o sinal filtrado.

\section{Medidas de Qualidade dos Algoritmos}

O SNR (Signal-to-Noise Ratio) é uma medida que relaciona um parâmetro (potência, energia ou variância) de um sinal $y=s+e$ com o parâmetro associado ao ruído $e$. Portanto, na avaliação do valor SNR é fundamental estimar esta componente de ruído de forma apropriada. Através da técnica de filtragem, busca-se ao mesmo tempo estimar $s$ (sinal sem ruído) e $e$ (ruído) e a avaliação da qualidade da componente $s$ dá-se através de medidas envolvendo a componente $e$ para o ruído estimado. Aqui, o parâmetro utilizado na análise é a variância $\sigma_{k}, k=y$ sinal e $k=e$ ruído, sendo $\bar{y}$ a média de $y$ e $\bar{e}$ a média de $e$. 


$$
S N R_{y}=10 \log _{10}\left(\frac{\sigma_{y}^{2}}{\sigma_{e}^{2}}\right), \quad \sigma_{y}^{2}=\frac{1}{N} \sum_{k=0}^{N-1}(y-\bar{y})^{2}, \quad \sigma_{e}^{2}=\frac{1}{N} \sum_{k=0}^{N-1}(e-\bar{e})^{2}
$$

Após a aplicação de um algoritmo de filtragem sobre um sinal ruidoso $y=s+e$, obtém-se uma estimativa do sinal $s$ desejado. Portanto, a componente de ruído $e$ é obtida subtraindo-se o sinal filtrado $s$ do sinal original $y, e=y-s$.

Para analisar a qualidade da filtragem através da relação sinal-ruído (SNR), calcula-se o SNR para o sinal filtrado (estimado) $s$. Como não é possível relacionar a variância do sinal $s$ com a variância de um possível ruído ainda presente em $s$, relaciona-se com a variância do ruído $e$, presente no sinal original $y, e=y-s$. Sendo assim, $S N R_{s}=10 \log _{10}\left(\frac{\sigma_{s}^{2}}{\sigma_{e}^{2}}\right)$.

Se a componete de ruído retirada for grande, o valor $\sigma_{e}^{2}$ tende também a ser elevado. Assim, quanto menor o valor do SNR, melhor a qualidade da filtragem. No entanto, se a filtragem for exagerada, o denominador de 15 continuará sendo grande, gerando um valor SNR ainda pequeno, mas que não mais indica qualidade alta da filtragem. Ou seja, filtragem eficiente ocorre quando o valor SNR encontrar-se em uma faixa de valores possíveis. E em cada aplicação esta faixa deve ser calibrada para que não se corra o risco de filtrar componentes relevantes para o sinal em análise.

Como os sinais cardíacos apresentam formas variadas, parâmetros como número de níveis na transformada wavelet direta, número de pontos do sinal analisado e frequiência de amostragem devem ser levados em consideração na aplicação dos algoritmos aqui propostos.

\section{Aplicações e Resultados}

Os testes numéricos nesta seção ilustram o potencial de filtragem dos algoritmos apresentados nas Seções 3 e 4 . A medida de qualidade utilizada na comparação das duas formulações é o SNR para a variância, amplamente utilizado na literatura [11]. De acordo com [10] cabe observar que medidas como o erro quadrático médio (MSE) podem mascarar perdas de propriedades relevantes para diagnóstico que ocorreram devido a uma filtragem excessiva. No entanto, avaliar a qualidade do sinal estimado utilizando MSE como uma das métricas ainda segue sendo considerado [14].

$\mathrm{Na}$ Figura 4(a) são apresentados os resultados de filtragem obtidos via algoritmos AFS - modificado e AFS sobre um sinal cardíaco sinusoidal sem patologias, digitalizado com frequência de amostragem de 200 amostras por segundo $\left(f_{a}=200 a / s\right)$, contendo ruído com SNR de $25 \mathrm{~dB}$ inserido através da soma de um ruído branco gaussiano. A transformada wavelet aplicada emprega 3 níveis de decomposição. Para os sinais com frequência de 
amostragem de 1000 amostras por segundo $\left(f_{a}=1000 a / s\right)$ foram considerados 8 níves da transformada wavelet. A escolha do número de níveis da transfomada depende basicamente de dois fatores: da resolução dos dados iniciais (frequência de amostragem inicial) e da resolução desejada no último nível da transformação [4], que depende da aplicação. No caso de ECG's há a preocupação em se preservar as ondas $\mathrm{P}$ e T após a filtragem, que podem ser descartadas se a fatoração wavelet permitir que níveis associados a frequências mais baixas sejam eliminados na filtragem. Isso limita a fatoração wavelet em uma quantidade menor de níveis do que a fatoração máxima [4, 5, 8, 9]. O complexo QRS e as ondas $\mathrm{P}$ e T são perfeitamente preservados nos sinais resultantes, como mostra a Figura4 As Figuras 4(b) e 4(c) apresentam o resultado da filtragem via algoritmos AFS - modificado e AFS para dois ECG's que apresentam uma certa arritmia cardíaca e com frequencias de amostragem distintas. Neste caso, o nível de ruído nestes sinais é de $25 \mathrm{~dB}$, inserido através da soma de um ruído branco gaussiano. Na Figura 4 (b), o sinal original possui $f_{a}=1000 a / s$ e na Figura 4(c) $f_{a}=200 a / s$. Na comparação entre a qualidade de filtragem para os dois algoritmos, cabe ressaltar que quanto menor o valor de SNR, melhor é a estimativa do ruído (filtragem), uma vez que preservam-se com mais intensidade as características de maior frequência do sinal, o que ocorre com as aproximações obtidas através do algoritmo proposto para diferentes frequências de amostragem. Em todas as aplicações foram utilizadas a transformada wavelet de Daubechies com 3 momentos nulos (Db3). A Tabela (1) apresenta os valores de SNR para os gráficos da Figura 4 Na Figura (5) é analisado um sinal normal original-

\begin{tabular}{|c|c|c|}
\hline Sinal & SNR(dB) Algoritmo AFS - Modificado & SNR(dB) Algoritmo AFS \\
\hline Figura 2(a) & 17.7513 & 22.9098 \\
\hline Figura 2(b) & 18.5814 & 18.7616 \\
\hline Figura 2(c) & 18.2121 & 25.5646 \\
\hline
\end{tabular}

Tabela 1. SNR do sinal filtrado estimado pelos algoritmos AFS-Modificado e AFS.

mente ruidoso da base de dados do MIT-BIH [6], cuja classificação é ltstdb - s20101. Sua frequência de amostragem é $f_{a}=1000 a / s$. Novamente a transformada wavelet direta (Db3) foi aplicada para 8 níveis de transformação. Pode-se observar que o formato do complexo QRS indicado na figura é preservado após a filtragem. A preservação dessa informação é importante tanto na classificação do sinal quanto na deteç̧ão de anomalias. A Tabela (2) apresenta os valores de SNR em dB para os 8 níveis de transformação apresentados na Figura (5). Como se pode observar nesta tabela, os valores de SNR obtidos a partir da aplicação do algoritmo AFS Modificado são menores em relação aos valores obtidos com o algoritmo AFS, resultando na remoção mais eficiente do ruído em todos os níveis de fatoração. A Figura 6 apresenta uma comparação de resultados de SNR para os dois algoritmos propostos neste trabalho com as técnicas soft e hard thresholding [2], amplamente empregadas como técnicas de filtragem de sinais cardíacos. Foram comparados os resultados para cinco níveis distintos de ruído do sinal orginal $(20 \mathrm{~dB}, 25 \mathrm{~dB}, 30 \mathrm{~dB}, 35 \mathrm{~dB}$ e $40 \mathrm{~dB})$ e empregados 8 níveis 

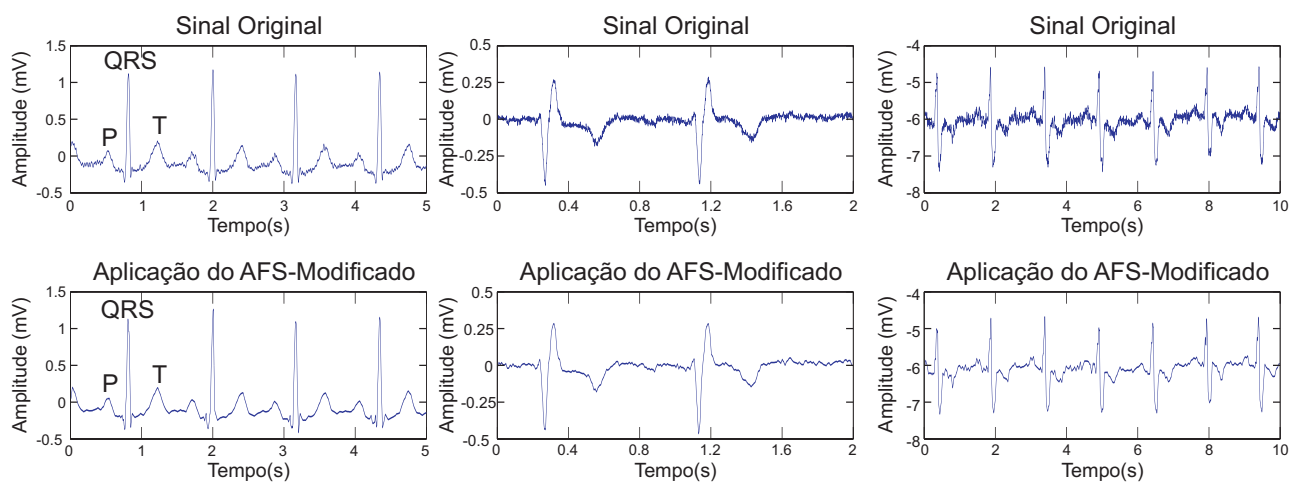

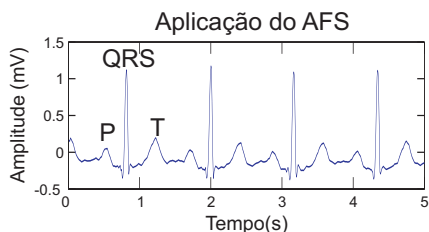

(a)

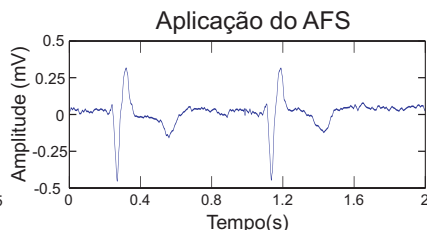

(b)

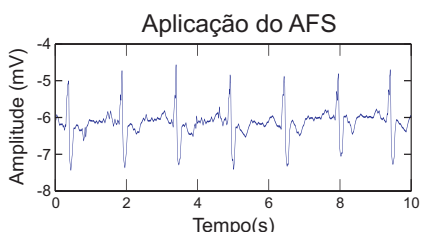

(c)

Figura 4. (a) Sinal cardíaco normal $y \operatorname{com} f_{a}=200 a / s$, (b) ECG com arritmia e $f_{a}=1000 a / s$, (c) ECG com arritmia e $f_{a}=200 a / s$.
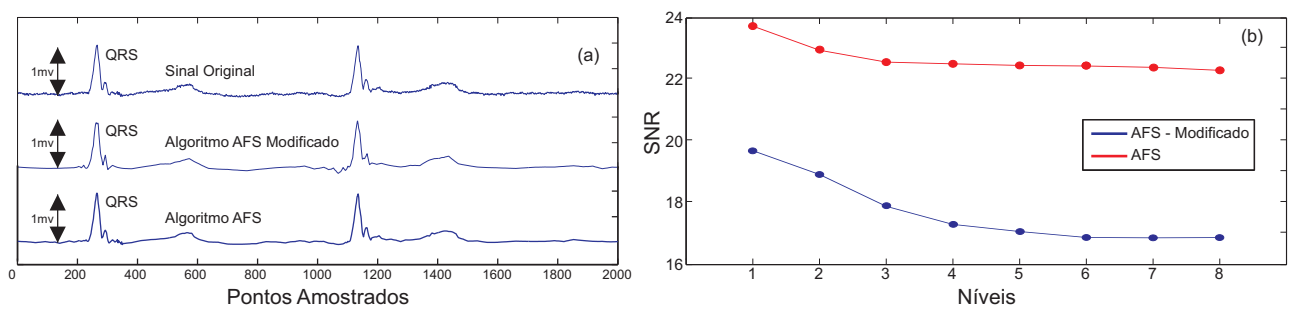

Figura 5. (a) Resultados dos algoritmos AFS Modificado e AFS para o oitavo e último nível de transformada. (b) Resultado da medida SNR para ambos os algoritmos para 8 níveis da transformada DB3.

de decomposição para a transformada wavelet. O SNR resultante para os algoritmos AFS Modificado e AFS são relativamente maiores que o SNR resultante da aplicação das técnicas de soft e hard thresholding. Apesar da medida de qualidade usada neste trabalho afirmar que um SNR baixo indica melhor qualidade de filtragem para sinais cardíacos, o fato do SNR 


\begin{tabular}{|c|c|c|c|c|c|c|c|c|}
\hline Níveis & 1 & 2 & 3 & 4 & 5 & 6 & 7 & 8 \\
\hline SNR(dB) AFS Modificado & 20.3067 & 19.3533 & 18.0792 & 17.3557 & 17.0694 & 16.8325 & 16.8179 & 16.8297 \\
\hline SNR(dB) AFS & 23.7536 & 22.9447 & 22.5467 & 22.4880 & 22.4340 & 22.4149 & 22.3709 & 22.2693 \\
\hline
\end{tabular}

Tabela 2. Razão Sinal-Ruído (SNR) para os algoritmos AFS-Modificado e recursivo AFS.

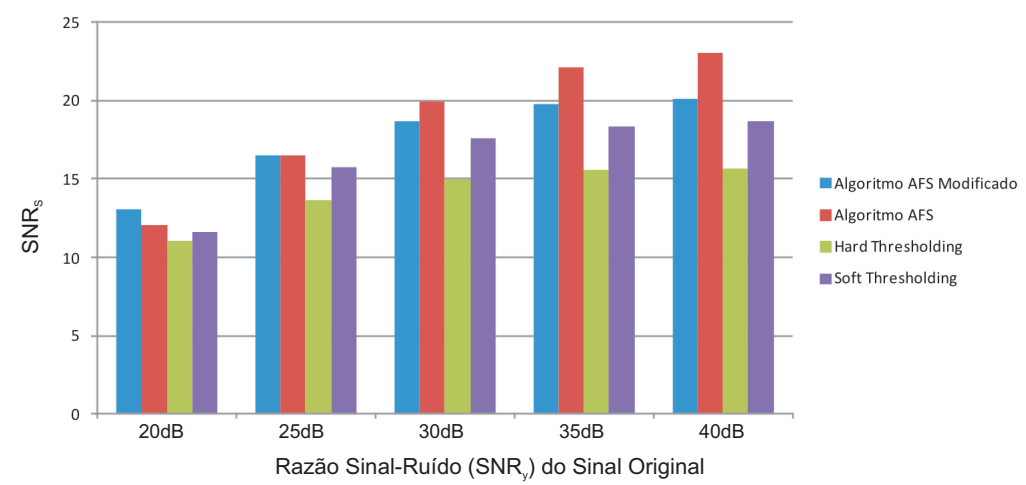

Figura 6. Comparação dos Algoritmos propostos com as técninas de Hard e Soft Thresholding.

resultante das técnicas de soft e hard thresholding ser muito mais baixo indica uma filtragem exagerada e deterioração das propriedades do sinal de ECG, como por exemplo, das ondas P e T e do complexo QRS.

\section{Conclusão}

Neste trabalho o algoritmo de Azzaline-Farge-Schneider, utilizado para análise de imagens, é adaptado para o caso de sinais unidimensionais e ainda é modificado para realizar a filtragem adaptativa de sinais cardíacos através da transformada wavelet ortonormal da família de Daubechies. A adaptividade é obtida uma vez que o limiar de corte para o truncamento de coeficientes wavelets da série do sinal é calculado através dos próprios valores a serem analisados.

Como o ruído é caracterizado por sua variância, este é um parâmetro importante na maior parte dos métodos de filtragem de sinais e imagens. Aqui, os algoritmos estimam a variância do ruído presente no sinal a partir dos coeficientes wavelets da série do sinal e o limiar de corte procurado depende desta variância. O algoritmo AFS Modificado (o primeiro 
apresentado) baseia-se em uma análise das frequências do sinal analisado para decidir quais níveis da transformação podem ser descartados, sem causar dano na reconstrução do sinal. Neste caso, o parâmetro de corte é calculado com base nas componentes da metade superior de espectro de frequências, que são informações consideradas como ruído do sinal.

O segundo (adaptação do algoritmo AFS) atua de maneira recursiva para estimar a variância do ruído, estabilizando ao longo do processo a quantidade de elementos retirados da série wavelet. Este processo, embora não tenha uma relação direta com aspectos fisiológicos associados aos sinais, estima satisfatoriamente o parâmetro associado ao ruído e com isso consegue evitar a remoção de informações importantes do sinal cardíaco ao longo da filtragem.

Os resultados para os dois algoritmos são apresentados através da relação sinal-ruído (SNR) tanto do sinal original, quanto do sinal filtrado. O algoritmo adaptativo AFS - Modificado proposto realiza a filtragem com poucas operações, sendo que a transformada wavelet rápida para $n$ níveis é realizada apenas uma vez, e o número de níveis $n$ dependente da frequência de amostragem inicial. O algoritmo recursivo necessita de um maior número de operações para filtrar o sinal e tende a manter um maior número de informações no processo de filtragem, fato explícito nos valores de SNR apresentados na Seção 6. A medida SNR baseada na variância foi utilizada como forma de avaliar o desempenho dos algoritmos quanto à qualidade do sinal estimado, comprovando a eficácea de ambos algoritmos propostos para a remoção de ruído, preservando as ondas $\mathrm{P}$ e $\mathrm{T}$ os complexos QRS, características essenciais do sinal analisado.

\section{Referências}

[1] A. Azzalini, M. Farge, e K. Schneider, Nonlinear wavelet thresholding; A recursive method to determine the optimal denoising threshold, Appl. Comput. Harm. Anal. 18 (2005), no. 2, 177-185.

[2] D. L. Donoho e I. M. Johnstone; Wavelet threshold estimators for data with correlated noise, J Royal Statist Soc, Vol. B 59 no. 2,(1997) 319-351.

[3] I. Daubechies; Ten lectures on wavelets, SIAM, Philadelphia, 1992.

[4] S. Mallat; A Wavelet Tour of Signal Processing, Third Edition: The Sparse Way 3rd edition, Academic Press, 2008 ISBN-10: 0123743702

[5] A. J. Kozakevicius, C. R. Rodrigues, R. C. Nunes, e R. Guerra Filho, Adaptative ECG filtering and QRS detection using orthogonal wavelet transform, (2006) Acta press, online.

[6] MIT-BIH data base, online: http://www.physionet.org/physiobank/database/mitdb. Acesso em 25 de Janeiro de 2009.

[7] O. M. Nielsen, Wavelets in scientific computing. Ph.D. Thesis, Technical University of Denmark, 1998. 
[8] L. Su e G. Zhao, De-Noising of ECG Signal Using Translation- Invariant Wavelet DeNoising Method with Improved Thresholding, Proceedings of the IEEE Engineering in Medicine and Biology 27th Annual Conference, China, September, 2005.

[9] X. Xu e Y. Liu, Adaptative Threshold for QRS Complex Detection Based on Wavelet Transform, Conf. Proc. IEEE Eng. Med. and Biol., 2005.

[10] Y. Zigel, A. Cohen e A. Katz, The Weighted Diagnostic Distortion (WDD) Measure for ECG Signal Compression, Proceedings of the IEEE, Vol. 47, No 11, November, 2000.

[11] M. Jansen, Wavelet Thresholding and Noise Reduction, Ph.D. Thesis, Katholieke Universiteit Leuven, 2000.

[12] Os princípios básicos do diagnóstico elétrico em cardiologia. http://www.misodor.com/ELETROCARDIOGRAMA.php (Acesso em 14 de Outubro de 2011).

[13] D. Dubin, Interpretação Rápida do ECG, Editora de Publicações Científicas, Rua das Américas, 1155.

[14] A. Kabir, C. Shahnaz; Comparisson of ECG signal denoising algorithms in EMD and wavelet domains. IJRRAS 11 (3), June 2012, pp. 499-516 